\title{
Dietary Practice and Associated Factors among Pregnant Women in Gondar Town North West, Ethiopia, 2014
}

\author{
Mekonnen Sisay Alemayehu ${ }^{1,}$, , Endalamaw Mengesha Tesema ${ }^{2}$ \\ ${ }^{1}$ Nutrition and Food Science Research Department, Ethiopian Public Health Institute, Addis Ababa, Ethiopia \\ ${ }^{2}$ Department of Public Health, Rift Valley University, Addis Ababa, Ethiopia
}

Email address:

mekudesu@gmail.com (M. S. Alemayehu),endx12@gmail.com (E. M. Tesema)

\section{To cite this article:}

Mekonnen Sisay Alemayehu, Endalamaw Mengesha Tesema. Dietary Practice and Associated Factors among Pregnant Women in Gondar Town North West, Ethiopia, 2014. International Journal of Nutrition and Food Sciences. Vol. 4, No. 6, 2015, pp. 707-712. doi: 10.11648/j.ijnfs.20150406.27

\begin{abstract}
Proper food and good nutrition are essential for survival, physical growth, mental development, performance and productivity, health and well-being. Pregnancy is a critical phase in a woman's life, when the expectant mother needs optimal nutrients of superior food qualities to support the developing fetus. The objective of this study was to assess the dietary practice and associated factors among pregnant women in Gondar town, Northwest Ethiopia. Community based cross sectional study was undertaken from March to April 2014 in Gondar town. The data were collected by using interviewer aided questionnaire and were analyzed using SPSS windows version (16.0). Multiple logistic regression was run to assess factors associated with the dependent variable at $P<0.05$ and to control the confounders. This study showed that good dietary practice was found to be $40.1 \%$ ( $95 \% \mathrm{CI}$ : $36.1-44.3 \%$ ) during pregnancy. Mothers education, monthly income, nutrition information and dietary knowledge had a positive significant with pregnant mothers' dietary practices $(P<0.001)$. As dietary practices of pregnant mothers were relatively low in this study, the government in collaboration and a strong integration with concerned bodies should be focused on providing nutritional education to increase the practices of pregnant mothers on maternal nutrition during pregnancy.
\end{abstract}

Keywords: Dietary Practice, Pregnant Mothers, Gondar Town, Ethiopia

\section{Introduction}

Malnutrition is one of the most serious health problem affecting children and their mothers in Ethiopia. Undernourished mothers face greater risks during pregnancy and childbirth, and their children set off on a weaker developmental path, both physically and mentally. Undernourished children have lower resistance to infection and are more likely to die from common childhood illnesses, such as diarrheal diseases and respiratory infections. Those who survive may be locked into a vicious cycle of recurring sickness and faltering growth, often with irreversible damage to their cognitive and social development. Malnutrition prevents individuals and even the whole country from achieving full potential, and is closely related with survival, poverty and development [1].

Nutrition throughout life has a major effect on health. This is true for pregnant women as adequate maternal nutrition is one of the best ways to ensure maternal and fetal wellbeing in developed and developing countries. A mother's nutritional status at conception, during pregnancy and lactation, plays a key role in determining her health and well-being, as well as that of her child. So does the quality and quantity of her diet. Providing a nourishing diet for pregnant and lactating women therefore results in significantly better infant health outcomes. Recent evidence suggests that it may also reduce the risk of chronic diseases later in life. During pregnancy and breast-feeding, the recommended intakes for most nutrients increase [2].

The ability of mother to provide nutrients and oxygen for her baby is a critical factor for fetal health and its survival. Failure in supplying the adequate amount of nutrients to meet fetal demand can lead to fetal malnutrition [3, 4]. The fetus responds and adapts to under nutrition but by doing so it permanently alters the structure and function of the body. Maternal over nutrition also has long-lasting and detrimental effects on the health of the offspring [4, 5].

Furthermore, evidences manifested that adequate intake of nutrition is a key component for individual's health and well-being, particularly during pregnancy. It is well 
documented that inadequate maternal nutrition results in increased risks of short term consequences such as; Intra Uterine Growth Restriction (IUGR), low birth weight, preterm birth, prenatal and infant mortality and morbidity [6].

It was commonly accepted that the fetus was nourished adequately at the expense of maternal stores and needs, however, it is becoming clear that this may not always be the case, and that fetal development can be less than optimal if certain nutrients are not available during intrauterine life [7].

Therefore the promotion of women's health and other preventive health care practice should start before birth, during intrauterine life and extends throughout different phases of their lives in order to sustain their general as well as their reproductive health [8].

In Ethiopia many studies have focused on nutritional status of pregnant women. Consistent evidence about Ethiopian's women dietary practice is lacking. Taking into consideration dietary practice that continue to occur throughout pregnancy, it will be informative to investigate and identify the dietary practice that occur among Ethiopian pregnant women and reasons associated with the dietary practice, to provide sufficient data for policy makers and planers in advising pregnant women on dietary practice and food intake.

Therefore, this study aimed to investigate dietary practice and associated factors of women during their pregnancy.

\section{Methods}

\subsection{Study Settings}

The study was conducted in Gondar town Northwest Ethiopia. It is located $727 \mathrm{~km}$ away from Addis Ababa, the capital city of Ethiopia and elevated at $2400 \mathrm{~m}$ above sea level. The town is administratively divided into 12 urban sub cities (comprising 23 kebeles). According to the Bureau of Finance and Economic Development report (BoFED) of 2012 the estimated population reside in Gondar town was 258,178, of whom $60,209(23.32 \%)$ were women of reproductive age group and $1850(0.49 \%)$ were pregnant mothers.

There are three hospitals (one referral, one defense and one private), eight health centers and two NGO clinics in the town which delivers health care services for the town community including pregnant mothers. The study was conducted from March to April, 2014.

\subsection{Study Design}

Community based cross sectional study was used to assess dietary practice and associated factors among pregnant women in Gondar town.

\subsection{Source Population}

The source population was all pregnant women who live in Gondar town.

\subsection{Study Population}

All pregnant women in the randomly selected clusters.

\subsection{Sample Size Determination}

In this study, sample size was determined by using single population proportion formula. Taking the prevalence of good dietary practice as $33.9 \%$ during pregnancyto obtain the maximum sample size with 5\% marginal error, 95\% CI, a none response rate of $10 \%$ and 1.5 design effect. Based on this, the actual sample size for the study was determined using the formula for single population proportion.

Therefore, from the above the sample size is:

$$
\mathrm{n}=\left(\mathrm{z}_{\alpha / 2}\right)^{2} \mathrm{p}(1-\mathrm{p}) / \mathrm{d}^{2}=\text { Dietary practice }(\mathrm{P}=33.9 \%) \mathrm{n}=344
$$

Thus, a minimum number of 344 pregnant women were the required number in the study. When we consider the design effect and a $10 \%$ none response rate, the minimum sample size required became: $344 * 1.5+(344 * 10 \%)=550$. The design effect considered was 1.5 by the assumption of homogeneity between study subjects in the clusters. Since cluster sampling technique was used, all pregnant mothers in the cluster were included in the study. Hence, the final sample size of this study became 580 .

\subsection{Sampling Procedure}

A cluster random sampling technique was applied. The town has 12 administrative urban sub cities (23 kebeles). Five sub cities were selected by simple random sampling from the total 12 sub cities (the smallest sampling unit). Then each sub city was considered as a cluster and all pregnant women during the study period in the selected clusters were included in the study. Pregnant women had been traced by health extension workers and with an initial short survey assessment.

\subsection{Data Collection Procedures}

Data was collected using structured interviewer administered questionnaire having two parts, the first part containing socio-demographic information. The second part of the questionnaire was about dietary practices. The questionnaire of practice on food and nutrition was used in this study.Scores of dietary practices were obtained by summation of each group of questions. Each question was given one mark if the answers were correct, favorable or healthy for dietary practice. Zero score was given if the responses were wrong, unfavorable or unhealthy for dietary practice questions.

Seven dietary practice questions were designed to assess practices of mothers on nutritionduring pregnancy. The score of the respondents has taken and respondents were classified as having good or poor dietary practices by taking their responses $>75 \%$ and below $75 \%$ respectively $[9,10]$.

Each study subject was visited from house to house in order to get their responses. Interviewers had introduced themselves and explain the purpose of the study using specific statements in a standard procedure. Consent to participate was obtained from each interviewee.

\subsection{Data Quality Control}

For administering the structured questionnaire, Four BSc and 10 diploma nurse professionals were employed as 
supervisor and data collector respectively. Training was given for two days on the objective, relevance of the study, confidentiality of information, respondent's right, time of data collection and reorganization of the collected data from respective sub cities and submission on due time.In addition pre-test was conducted on $5 \%$ of the actual sample size.

\subsection{Variables}

\subsubsection{Dependent Variable}

Dietary practice of pregnant women.

\subsubsection{Independent Variables}

- Socio demographic and socio economic factors: Age, Residence, Marital status, Religion, Educational level, Occupation, Income level.

- Dietary information and gravidity: Nutrition education, Family size, Gap between pregnancy, Number of pregnancies

- Dietary knowledge of pregnant women

- Dietary attitude of pregnant women

\subsection{Data Processing and Analysis}

Data were checked, coded and entered to Epi-info version 3.5.3 and was exported to SPSS (Statistical Package for Social science) version 20 for analysis. Data entry was made by the principal investigator. Descriptive and analytical statistics including univariable, bivariable and multivariables analysis were employed. Bivariable analysis was used to examine association between dependent and independent variables. All variables with $\mathrm{p}<0.2$ in bivariable analysis were inserted in to the multiple logistic regression model to identify factors independently associated with dietary practice of pregnant women. Forward stepwise likelihood ratio was used to select the final independent predictors.

Significance was obtained at Odds ratio with 95\% CI and $\mathrm{p}<0.05$.Hosmer and lemeshow goodness of fit test result for the final model was done $(\mathrm{P}$. value $=5.89)$.

\subsection{Ethical Consideration}

Ethical clearance was obtained from Ethical review committee of University of Gondar. In order to obtain permission letter, we made contact Gondar town health office. Then, the pregnant women from each selected clusters were informed about the purpose of the study, the importance of their participation, and the right to withdraw at any time. And verbal consent was obtained prior to data collection. Privacy and confidentiality of information given by each respondent kept properly and name was not recorded. And the recorded data was kept in a secured place with strict confidentiality.

\section{Results}

\subsection{Socio-demographic Characteristics}

The survey included 580 individuals, among these 574 responded to the questionnaire and making the response rate of $(98.97 \%)$.
The mean age $( \pm \mathrm{SD})$ of the participants was $26.65( \pm 4.91)$ years. Considerably high proportions of the respondents $(46.2 \%)$ were in the age range of $25-34$ years. Besides, most study respondents were married (90.9\%).

Regarding the ethnic composition and religion of the respondents about greater than three quarter of them were Amhara and the majority of respondents' religion was Orthodox followed by Muslim followers.

Table 1. Socio demographic characteristics of pregnant women in Gondar town, Northwest Ethiopia, $2014(n=574)$.

\begin{tabular}{|c|c|c|}
\hline Variable & Number & Percent (\%) \\
\hline \multicolumn{3}{|l|}{ Age } \\
\hline $15-24$ & 197 & 34.3 \\
\hline $25-34$ & 265 & 46.2 \\
\hline $35-41$ & 112 & 19.5 \\
\hline \multicolumn{3}{|l|}{ Marital status } \\
\hline Married & 522 & 90.9 \\
\hline Single & 20 & 3.5 \\
\hline Separated/Divorced/Widowed & 32 & 5.6 \\
\hline \multicolumn{3}{|l|}{ Ethnicity } \\
\hline Amhara & 532 & 92.7 \\
\hline Tigre & 29 & 5.1 \\
\hline Oromo/ Gurage & 13 & 2.3 \\
\hline \multicolumn{3}{|l|}{ Religion } \\
\hline Orthodox & 437 & 76.1 \\
\hline Muslim & 129 & 22.5 \\
\hline Protestant & 8 & 1.4 \\
\hline \multicolumn{3}{|c|}{ Educational status of pregnant mothers } \\
\hline No formal education & 115 & 20 \\
\hline Primary level $\left(1-8^{\text {th }}\right)$ & 160 & 27.9 \\
\hline Secondary level $\left(9-12^{\text {th }}\right)$ & 200 & 34.8 \\
\hline Diploma and above & 99 & 17.2 \\
\hline \multicolumn{3}{|l|}{ Husband educational status } \\
\hline No formal education & 85 & 14.8 \\
\hline Primary level $\left(1-8^{\text {th }}\right)$ & 135 & 23.5 \\
\hline Secondary level $\left(9-12^{\text {th }}\right)$ & 182 & 31.7 \\
\hline Diploma and above & 141 & 24.6 \\
\hline \multicolumn{3}{|l|}{ Monthly income } \\
\hline$<1000$ birr & 248 & 43.2 \\
\hline $1000-2000$ birr & 189 & 32.9 \\
\hline$>2000$ birr & 137 & 23.9 \\
\hline \multicolumn{3}{|l|}{ Employment status } \\
\hline House wife & 297 & 51.7 \\
\hline Employed & 106 & 18.5 \\
\hline Unemployed & 74 & 12.9 \\
\hline Business & 97 & 16.9 \\
\hline \multicolumn{3}{|l|}{ Family size } \\
\hline $1-2$ & 184 & 32.1 \\
\hline $3-5$ & 263 & 45.8 \\
\hline $6+$ & 127 & 22.1 \\
\hline \multicolumn{3}{|l|}{ Nutrition information } \\
\hline Yes & 448 & 78 \\
\hline No & 126 & 22 \\
\hline \multicolumn{3}{|c|}{ Number of pregnancies before current pregnancy } \\
\hline$<2$ & 398 & 69.3 \\
\hline $3-5$ & 154 & 26.8 \\
\hline $5+$ & 22 & 3.8 \\
\hline \multicolumn{3}{|c|}{ Gap duration between past and current pregnancy } \\
\hline$<2$ years & 244 & 42.5 \\
\hline $2-5$ years & 287 & 50.0 \\
\hline $5+$ years & 43 & 7.5 \\
\hline
\end{tabular}

Concerning educational level, about $115(20 \%)$ of the respondents were illiterate and 200 (34.8) were secondary level education completed. Concerning the occupation of 
respondents, about half (51.7\%) were house wives and 109 (18.5\%) were employed (Table 1$)$.

Regarding pregnant women's estimated monthly income about 248 (43.2\%) earned less than 1000 birr per month, 189 $(32.9 \%)$ of them earned 1000-2000 birr per month and 137 $(23.9 \%)$ of the respondents had monthly income greater than 2000 birr.Among study participants 263 (45.8\%) had 3-5 family size, $187(32.1 \%)$ had $1-2$ family size and the remaining $127(22.1 \%)$ had 6 or more family size (Table 1$)$.

Four hundred seventeen $(72.6 \%)$ of the respondents had nutritional information during their pregnancy. Regarding their number of pregnancies, 398 (69.3\%), 154 (26.8\%), 22 $(3.8 \%)$ of the respondents had $<2,3-5$ and 5 or more number of pregnancies including the present one respectively.Among the total respondents, about half $287(50.0 \%)$ had also 3-5 years of gap duration between their pregnancies (Table 1).

\subsection{Dietarypractices of Pregnant Mothers During Pregnancy}

Out of 574 study participants responded for the questions to assess their dietary practices, 59.9 of them had poor dietary practice in which, less than half 229 (39.9\%) respondents had practiced avoiding food during their pregnancy. Out of those who avoided food during their pregnancy, $(46.7 \%)$ reported, it makes the baby big, (36.0\%) reported culture, (14.7\%) reported, it makes delivery difficult and $(2.6 \%)$ reported religions. But the rest, $345(60.1 \%)$ of the respondents had not avoided any food item during their pregnancy.

Four hundred twenty three $(73.5 \%)$ and $371(64.6 \%)$ of the respondents had practiced the habit of eating snacks and carbohydrates between meals during their pregnancy respectively. However about 151 (26.5\%) and 203 (35.4\%) of the respondents did not practiced eating snacks and carbohydrate between meals during their pregnancy.

Concerning the diet frequency of meal per day, about 89 (15.5\%) of the respondents had diet frequency of meal 1-2 per day during their pregnancy. The majority of pregnant mothers, $445(77.5 \%)$ had meal frequency of 3-4 times per day. The rest, $40(7.0 \%)$ had diet frequency of meals $>5$ per day during their pregnancy.Regarding to weight monitoring during their pregnancy most of the respondents, $477(83.1 \%)$ had practiced in following their weight during pregnancy whereas the rest $97(16.9 \%)$ of the respondents had not.

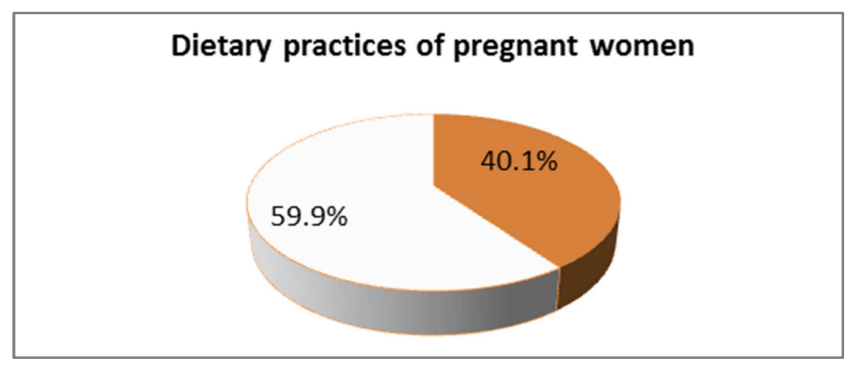

Figure 1. Distribution of dietary practice among pregnant mothers in Gondar town, Northwest Ethiopia, $2014(n=574)$.

From the general questions offered to the respondents to assess their dietary practice only $40.1 \%$ (95\% CI: $36.1-44.3 \%)$ of pregnant mothers found to have good dietary practice during their pregnancy, (Fig 1).

\subsection{Factors Associated with Dietary Practice of Pregnant Mothers}

Bivariate analysis showed that there was statistically significant association between dietary practices of mothers and monthly income, mother's educational status,knowledge, attitude, husband educational status and nutrition information, whereas, age, marital status, family size, number of pregnancies and gap between pregnancies had no association with dietary practices of mothers.

Multivariate analysis showed that there was statistically significant association between family income and dietary practices of mothers. The likelihood of dietary practice during pregnancy among mothers who had monthly income of 1000-2000 was 2 times higher than those $<1000$ monthly income $(\mathrm{AOR}=2.18,95 \% \mathrm{CI}: 1.39,3.39)$.

This studyalso identified that educational status had strong statistical association with dietary practices of mothers during pregnancy. Relative to the pregnant women with no formal educationwomen with educational level ofdiploma and above had nearly 3 times more likely to hadgood dietary practice during pregnancy $(\mathrm{AOR}=2.59,95 \% \mathrm{CI}: 1.38-4.85)$.

Table 2. Factors associated with dietary practice among pregnant women in Gondar town, Northwest Ethiopia, 2014. $(n=574)$.

\begin{tabular}{|c|c|c|c|c|}
\hline Variables & PracticeGood & Poor & $\begin{array}{l}\text { COR with } 95 \% \\
\text { CI }\end{array}$ & $\begin{array}{l}\text { AOR with } 95 \% \\
\text { CI }\end{array}$ \\
\hline \multicolumn{5}{|c|}{ Monthly income } \\
\hline$<1000$ & 64 & 184 & 1.00 & 1.00 \\
\hline $1000-2000$ & 97 & 92 & $3.03(2.03,4.54)$ & $2.18(1.39,3.41)$ \\
\hline$>2000$ & 69 & 68 & $2.92(1.88,4.53)$ & $1.59(0.97,2.63)$ \\
\hline \multicolumn{5}{|c|}{ Women's education } \\
\hline $\begin{array}{l}\text { No formal } \\
\text { education }\end{array}$ & 27 & 88 & 1.00 & 1.00 \\
\hline $\begin{array}{l}\text { Primary } \\
\left(1-8^{\text {th }}\right)\end{array}$ & 58 & 102 & $1.85(1.08,3.18)$ & $1.7(0.98,2.94)$ \\
\hline $\begin{array}{l}\text { Secondary } \\
\left(9-12^{\text {th }}\right)\end{array}$ & 93 & 107 & $2.83(1.70,4.73)$ & $2.54(1.50,4.3)$ \\
\hline $\begin{array}{l}\text { Diploma \& } \\
\text { above }\end{array}$ & 52 & 47 & $3.61(2.01,6.47)$ & $2.59(1.38,4.85)$ \\
\hline \multicolumn{5}{|c|}{ Nutrition information } \\
\hline Yes & 204 & 244 & $3.22(2.01,5.14)$ & $2.39(1.44,3.97)$ \\
\hline No & 26 & 100 & 1.00 & 1.00 \\
\hline \multicolumn{5}{|l|}{ Knowledge } \\
\hline Yes & 161 & 142 & $3.32(2.33,4.73)$ & $2.32(1.56,3.43)$ \\
\hline No & 69 & 202 & 1.00 & 1.00 \\
\hline \multicolumn{5}{|l|}{ Attitude } \\
\hline Positive & 145 & 186 & $2.35(1.62,3.39)$ & $1.22(0.83,1.78)$ \\
\hline Negative & 85 & 158 & 1.00 & 1.00 \\
\hline
\end{tabular}

Note; $1.00=$ Reference, p. value $=<0.05$

Moreover dietary knowledge had displayed significant positive relationship with dietary practices of mothers during pregnancy. Relative to women, who were not knowledgeable on diet, women who were knowledgeable had significantly more odds of good dietary practices during pregnancy $(\mathrm{AOR}=2.32,95 \% \mathrm{CI}: 1.56,3.43)$. From further multivariable 
analysis, the finding of the study identified that information about nutrition during pregnancy had strong statistical association with dietary practices of mothers during pregnancy. Relative to the pregnant women who had no nutrition information during pregnancy, women with nutrition information were nearly 2.4 more likely to had good dietary practice during their pregnancy $(\mathrm{AOR}=2.39,95 \% \mathrm{CI}$ : 1.44-3.97), (Table 2).

\section{Discussion}

Adequate nutritional intake is one of the most important factors affecting on one's health and well-being, especially during pregnancy [11].

$\mathrm{Up}$ on the general questions offered to the respondents to assess their dietary practice only $40.1 \%$ of pregnant mothers found to have good dietary practice during their pregnancy.

This figure is lower than the study conducted in America in which the resultsof the study revealed that more than half of women $(54 \%)$ had a poor level of knowledge and practices regarding the intake of essential nutrients and basic food elements meeting the nutritional demands of the mother and the fetus [9].

The results of the present study also showed lower dietary practices of mothers compared to that of Malaysia study in that the mean (SD) score for nutrition attitude and practices was $14.9 \pm 2.6$ and $3.7 \pm 0.8$ which presented a $57.3 \%$ and $74 \%$ correct response rate of the pregnant women had good practices on nutrition during pregnancy respectively [12].

This low dietary practice of pregnant mothers may be due to their low income, relatively high family size, lack of nutritional information and low educational status of the study participants as compared to other previous studies.

Comparing the present study to that conducted in Pakistan, the present one is lower than the previous study which, revealed that despite having the knowledge on nutrition during pregnancy and lactation ( $84 \%$ women) only $65.5 \%$ put it in practice during their pregnancy [13].

The result of the present study was higher than the study conducted in rural district community in Oromiya region in Ethiopia in which the good dietary practice of pregnant mothers were $33.9 \%$ [14].

The discrepancy of the two studies may be due to the fact that the differences between the study participants, in that the present study was conducted on urban communities ( pregnant mothers) which can be better than rural mothers in terms of educational, economic status and more access to nutrition information during pregnancy.

The finding of this study identified that educational status have strong statistical association with dietary practices of mothers during pregnancy $(\mathrm{AOR}=2.59,95 \% \mathrm{CI}$ : 1.38-4.85). Which is supported by the study conducted in America in which women with high level of education had high mean (\%) of their nutritional knowledge and practice compared to low and moderate educational level [9].

In addition the finding of this study revealed that monthly income has statistical association with dietary practices of mothers during pregnancy $(\mathrm{AOR}=2.18, \mathrm{CI}: 1.39,3.39)$.

Similar findings had also been reported from a study in Brazil that identified higher educational level and income had significant association with dietary practice of pregnant mothers [15].

The present study also demonstrated that there was statistical association between information about nutrition and dietary practices during pregnancy $(\mathrm{AOR}=2.39$, CI: 1.44-3.97). This result is supported by the study conducted in Oromiya region in which nutrition information was the predictor factor for dietary practices in the study area $(\mathrm{AOR}=6.264$, CI: 3.487-11.254) [14].

Moreover in this study women's dietary knowledge had shown positive relationship with dietary practice of mothers during pregnancy $(\mathrm{AOR}=2.32,1.56-3.43)$. This finding is also in agreement with the study conducted in America in which the most significant predictor for good dietary practice was women's dietary knowledge $(\mathrm{t}=3.315, p<0.05)$ [9].

In this study husband educational status, age, marital status, gap duration between pregnancy, family size and employment status and number of pregnancies were not associated with dietary practice of mothers during their pregnancy.

\section{Conclusion}

From the present study, it can be concluded that, majority of pregnant mothers $(59.9 \%)$ had a poor dietary practice during pregnancy. They lacked the basic and the essential practice to consume vegetables, fruits, egg and others which are the basic sources of most of the types of vitamins and minerals.Moreover, unhealthy food practice was observed among them.

The current study also showed that, the most significant predicting factors for practice in this study were women's nu knowledge, nutrition information, monthly income and educational status. Therefore it is obvious that good knowledge, nutrition education about basic nutrients and adequate and balanced diet usually resulting in positive dietary practices which are important determinants of optimum health from conception until death.

\section{References}

[1] IYCN. Infant \& Young Child Nutrition ProjectLiterature Review Prepared for the Message and Materials Development Workshop produced through support provided by the United States Agency for International Development (USAID), Addis Ababa, Ethiopia. January 2011.

[2] Barker et al. Maternal Nutrition from pre-pregnancy to lactation. The Lancet (1993);341 (8850):938-41.

[3] L MKW. Aboriginal teenage pregnancies compared with non-aboriginal in South Australia. JObstet Gynecol. 2003;42(2) 187-92.

[4] Silva AM STSM. Maternal nutrition as a determinant of birth weight. Achieves of Disease in Childhood. 2003;86(1):4-6. 
[5] Jenson j. SL, Mary B. Nutrition science \& application, 3rd. Tokyo. Saunders Collage publishing. 2003:35-63.

[6] Luigi R, et al. Effects of pregnancy on eating attitudes and disorders. A prospective study. Journal of Psychosomatic Research. (2005);59 (3): 175 -9.

[7] Z S. Is maternal diet supplementation beneficial? Optimal development of infant depends on mother's diet. American Journal of Clinical Nutrition 2009.

[8] Excellence NIfHaC. Improving the health and nutrition of pregnant and breastfeeding mothers and children in low-income households.2009, August 22.

[9] Shehab L. Nutritional Awareness of Women during Pregnancy. Journal of American Science. 2012;8(7).

[10] Mitra Mirsanjari WA. Dietary Knowledge and Behaviors in a Sample of Malay Pregnant Women. UMT 11th International Annual Symposium on Sustainability Science and Management, Terengganu, Malaysia. 09th - 11th July 2012.

[11] E.M. Szwajcer GJH, M.A. Koelen, C.M.J Van Woerkum. Nutrition awareness and pregnancy, Implications for the life course perspective. European Journal of Obstetrics \& gynecology Reproductive Biology. 2006; 135 (1): 58-64.
[12] Mitra Mirsanjari WAMWM, Affizal Ahmad, Mohd Shukri Othman and Maryam Mosavat. Does Nutritional Knowledge Have Relationship With Healthy Dietary Attitude and Practices during Pregnancy? International Conference on Nutrition and Food Sciences, (C) (2012) IACSIT Press, Singapore. 2012;39.

[13] Shahid Mahrnood MFA SS, Azhar Mujeeb, Naira Bano, Humaira Mubasher, Department of Community Medicine, Allama Iqbal Medical College, Lahore. Assessment of Nutritional Beliefs and Practices in Pregnant and Lactating Mothers in an Urban and Rural Area of Pakistan. 60-2.

[14] Gemeda Daba FB, Wondu Garoma and Habtamu Fekadu. Assessment of Nutritional Practices of Pregnant Mothers on MaternalNutrition and Associated Factors in Guto Gida Woreda, East Wollega Zone, Ethiopia. Science,Technology and Arts Research Journal. July-Sep 2013; volume 2(3): 105-13.

[15] Juliana Feliciati Hoffman ea. Dietary patterns during pregnancy and the association with sociodemographic characteristics among women attending general practices in southern Brazil the ECCAGe Study. Cadernos de Saúde Pública. May 2013; 29 no.5. 